\title{
Kinetics of water adsorption on minerals and the breathing of the Martian regolith
}

\author{
P. Beck, ${ }^{1}$ A. Pommerol, ${ }^{1}$ B. Schmitt, ${ }^{1}$ and O. Brissaud ${ }^{1}$ \\ Received 6 November 2009; revised 5 July 2010; accepted 22 July 2010; published 21 October 2010.
}

[1] Several observations of the total amount of water vapor in Mars atmosphere display diurnal variations. A possible explanation is an atmosphere/surface coupling that occurs through $\mathrm{H}_{2} \mathrm{O}$ exchange with the regolith, where adsorbed water molecules have been proposed as a consequent water reservoir. In order to test this hypothesis, experimental laboratory measurements of adsorption isotherms are needed together with adsorption kinetics measurements. Following our previous measurements of the adsorption isotherms of a series of Mars surface analog materials, we report here on kinetics measurements on the same samples at a temperature of $243 \mathrm{~K}$ (volcanic tuff, dunite, ferrihydrite, smectite, JSCMars1). We observed that even for thin samples $(1 \mathrm{~mm})$, diffusion through the sample might influence the adsorption process and significant caution is required to infer kinetics parameters of strongly adsorbing samples. The kinetics parameters $k_{d}$ and $d k_{a} / d P$ were extracted following the Langmuir theory. Results show that adsorption is fast but not instantaneous with regard to the diurnal time scale $\left(k_{d}=10^{-2}-10^{-3} \mathrm{~s}^{-1}, d k_{a} / d P=10^{-3}-10^{-4}\right.$ $\left.\mathrm{Pa}^{-1} \mathrm{~s}^{-1}\right)$. Large variations are found between the different samples, which suggest a possible geological control on the amount of exchangeable water between the regolith and the atmosphere. We estimate the impact of a noninstantaneous kinetics on the diurnal water vapor cycle by calculating the maximum amount of exchangeable water. We found that a significant amount of $\mathrm{H}_{2} \mathrm{O}$ can be trapped within the regolith, even in weakly adsorbing analog materials. The similarity in adsorption properties between the JSCMars1 and ferrihydrite samples suggests that the adsorption properties of the latter are controlled by the presence of iron oxyhydroxide. These materials have strong adsorption capacities, and their presence on the Martian surface might explain the observed spatial correlation between the average surface humidity and the abundance of surface dust.

Citation: Beck, P., A. Pommerol, B. Schmitt, and O. Brissaud (2010), Kinetics of water adsorption on minerals and the breathing of the Martian regolith, J. Geophys. Res., 115, E10011, doi:10.1029/2009JE003539.

\section{Introduction}

[2] Both spacecraft and ground-based observations have suggested the presence of a diurnal cycle in the atmospheric water abundance on Mars [Jakosky et al., 1988; Melchiorri et al., 2009; Smith, 2002; Sprague et al., 2003, 2006]. In certain localities, minima are observed in the early morning and late afternoon with respect to high noon abundance. The total amount of water in the atmospheric column can vary by 10-20 precipitable microns during the course of the day [Melchiorri et al., 2009; Sprague et al., 2003], an enrichment that was proposed to reflect $\mathrm{H}_{2} \mathrm{O}$ exchange with the surface/subsurface. A number of studies have tried to reproduce these variations from numerical models of the water transfer through an adsorbing regolith [Bottger et al.,

\footnotetext{
${ }^{1}$ Laboratoire de planétologie de Grenoble, Université Joseph Fourier OSUG, Grenoble, France.
}

Copyright 2010 by the American Geophysical Union. 0148-0227/10/2009JE003539
2005; Zent et al., 1993, 2001] but failed in reproducing the observations.

[3] In order to model successfully the "breathing" of the regolith, several parameters need to be experimentally determined for analog materials of the Martian surface [Bryson et al., 2008; Chevrier et al., 2008; Janchen et al., 2006, 2009; Zent et al., 2001; Zent and Quinn, 1995, 1997]. The so-called "adsorption isotherms" characterize the amount of a given molecule $\left(\rho_{\mathrm{a}}\right.$ in $\left.\mathrm{kg} \mathrm{m}^{-3}\right)$ that is adsorbed when at equilibrium with a given vapor partial pressure. In the Langmuir kinetics theory, the isotherm has two parameters, the $C$ constant (which relates to the specific surface area) and the $\alpha$ parameter that characterizes the pressure dependence of $\rho_{\mathrm{a}}$ [Gregg and Sing, 1982]. Lacking kinetics measurements of adsorption at relevant $P$ and $T$, all regolith models suppose an instantaneous process which is parameterized by empirical adsorption isotherms measured in the laboratory. However, a significant time lag exists in order to reach equilibrium due to an "intrinsic" kinetics of the adsorption process. This intrinsic kinetics is the time required for a single homogeneous particle (SHP) to reach 


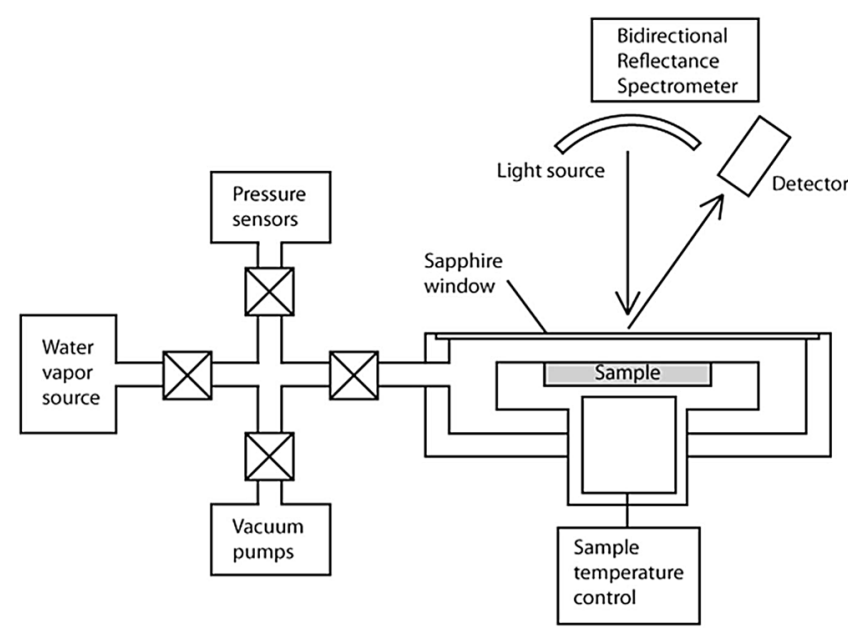

Figure 1. Schematic of the experimental setup used in this study and Pommerol et al. [2009].

its equilibrium surface coverage, and is usually poorly constrained for geological materials due to the highly challenging deconvolution of adsorption and diffusion kinetics during the experiment.

[4] This is a follow-up paper to the article published by Pommerol et al. [2009]. Here, we use the previously measured isotherms of five different Martian soil analogs to infer the transfer properties of the Martian subsurface by physical calculations. In parallel, using near-infrared reflectance spectroscopy, we measured intrinsic kinetics of water adsorption for the same materials, which sheds light on the possible time scale for subsurface/atmosphere exchange of water, and the volume of exchangeable water involved in the diurnal and seasonal cycles.

\section{Methods and Samples Description}

[5] All the laboratory measurements presented and discussed in this article have been obtained together with those reported by Pommerol et al. [2009] and a schematic of the experimental setup, reproduced from this article, is presented in Figure 1. While spectral and thermodynamic parameters from the latter article were always obtained at equilibrium, the current study is devoted to the characterization of the kinetics of water exchange between each of the equilibrium states. This section briefly describes the methods and samples used for this study. Many more details about the experimental setup and procedures as well as a complete description of the samples are provided by Pommerol et al. [2009].

\subsection{Samples Nature and Preparation}

[6] Five different types of materials were studied. All of these materials are either suspected to be major mineral components of the Martian regolith or are seen as good analogs of Martian surface materials. Those samples have been extensively described by Pommerol et al. [2009] and Pommerol and Schmitt [2008a, 2008b]. They are the JSC Mars 1 palagonitic soil, ferrihydrite, a volcanic tuff of basaltic composition, a sodic smectite ( $\mathrm{SWy}-2)$ and a dunite ( $>90$ wt. \% olivine) powder. In the case of the dunite, the smectite and the volcanic tuff, we used sieved particle size fractions with grain diameters ranging between 25 and $50 \mu \mathrm{m}$. For each sample, specific surface area determined from water vapor adsorption can be found in the work of Pommerol et al. [2009].

[7] Sample holder (diameter, $30 \mathrm{~mm}$; thickness, $1 \mathrm{~mm}$ ) is filled to rim with the noncompacted sample powder and then flattened with a spatula to obtain a smooth surface without powder compaction. In these conditions, mass of the samples ranges between 500 and $900 \mathrm{mg}$ depending on the minerals density and material porosity.

[8] The sample holder filled with the mineral powder is then placed in the simulation chamber (see section 2.2). The dehydration process is divided in successive steps that aim to remove the maximum of moisture from the sample while avoiding deterioration of its surface flatness (volume change and dehydration cracking). First, the sample is heated at $443 \mathrm{~K}$ following a temperature ramp of $1 \mathrm{~h}$. After $1 \mathrm{~h}$ of heating at $443 \mathrm{~K}$, chamber is closed and heating is stopped. Sample temperature decreases during a few hours to reach the temperature of the cold room $(263 \mathrm{~K})$. Then, the simulation chamber is slowly pumped down to pressure lower than $10^{-6}$ mbar. Under secondary vacuum, the sample is heated again at $443 \mathrm{~K}$ during a minimum of $10 \mathrm{~h}$. In the same time, the chamber itself and fittings are heated using heating cords to ensure a quick and efficient outgassing. Finally, the sample is cooled to the temperature at which measurements are performed $(243 \mathrm{~K})$ and temperature is then kept constant $( \pm 0.1 \mathrm{~K})$ for the complete set of measurements.

\subsection{Environmental Chamber}

[9] We designed and built an environmental chamber in relation with an adsorption setup as a complement to the LPG spectrogonio radiometer (see description below and Figure 1). Extensive description of the chamber as well as a discussion of the uncertainties on the measurements are provided by Pommerol et al. [2009].

[10] The temperature of the sample is adjusted and controlled using a heating resistance, a thermoelectric cooler and a Pt thermocouple placed at the bottom of the sample. All measurements presented in this paper were made at a constant temperature of $243 \mathrm{~K}$. A 1 Torr MKS Baratron ${ }^{\circledR}$ absolute pressure sensor, which measures absolute pressure value between $10^{-6}$ and $1 \mathrm{mbar}$, is used to monitor pressure inside the chamber. The source of water vapor is a volume of ultra pure, dematerialized and carefully outgassed liquid water maintained at a temperature of $293 \mathrm{~K}$.

[11] The chamber is closed on its upper surface by a sapphire window, which has an excellent transmission and is spectrally featureless in the visible and near infrared. The optical path within the chamber is of a few $\mathrm{cm}$ and the spectral signatures of $\mathrm{H}_{2} \mathrm{O}$ vapor within the chamber are negligible. Minor contribution of $\mathrm{H}_{2} \mathrm{O}$ and $\mathrm{CO}_{2}$ vapor can be present in the 2.6-2.8 $\mu \mathrm{m}$ range due to the cold room atmosphere [Pommerol et al., 2009, Figure 13] but gas absorption does not interfere with any of the spectral features of adsorbed $\mathrm{H}_{2} \mathrm{O}$ that are used in the present study. Potential adsorption of water elsewhere than on the sample would also induce a severe bias in the retrieval of the quantity of adsorbed water in the sample and on adsorption kinetics. As a test, we measured the adsorption isotherm of the empty chamber without any sample. Under appropriate conditions (low vapor relative pressure and reasonably adsorbent sample), the quantity of 

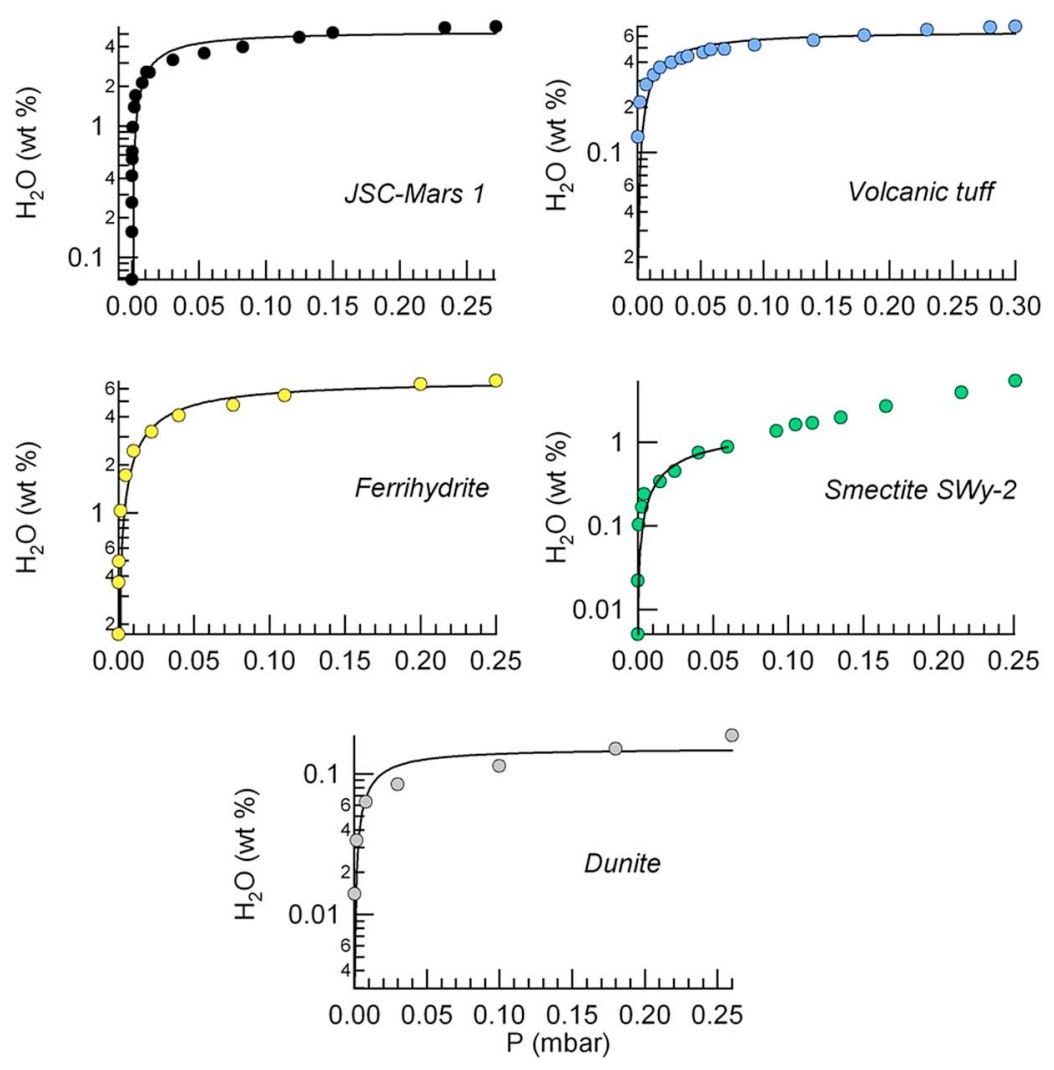

Figure 2. Adsorption isotherm for the five samples studied here. The amount of adsorbed water is plotted as a function of relative pressure $\left(P_{r}=P / P_{\text {sat }}\right.$, with $P_{\text {sat }}$ the water vapor saturation pressure). The data are from Pommerol et al. [2009] and were fitted according to the Langmuir theory (solid lines).

water adsorbed on the various interior surfaces of the setup was found to be negligible. Using a combination of spectroscopic and thermodynamic information, we can calculate accurately the amount of adsorbed water for a wide range of relative humidity (see section 2.5 ).

\subsection{Reflectance Spectrometer}

[12] The LPG spectrogonio radiometer [Brissaud et al., $2004]$ is installed in a cold room at a temperature of $263 \mathrm{~K}$. Sample spectra are measured relative to commercial reference surfaces: Spectralon ${ }^{\circledR}$ (Labsphere Inc.) for the spectral range 0.4-2.5 $\mu \mathrm{m}$ and Infragold ${ }^{\mathbb{B}}$ (Labsphere Inc.) for the spectral range $2.5-4.8 \mu \mathrm{m}$. Corrections are applied to take into account minor absorptions in the Spectralon ${ }^{\circledR}$ spectrum and non-Lambertian behaviors of the Spectralon ${ }^{\circledR}$ and Infragold ${ }^{\circledR}$ surfaces [Bonnefoy, 2001]. We used the spectrometer to perform continuous measurements (1 value per second) of the sample reflectance at one particular wavelength inside the absorption bands of adsorbed water: either 3.10 or $1.93 \mu \mathrm{m}$.

\subsection{Experimental Procedure}

[13] In the initial state, the entire setup is under static high vacuum $\left(P<10^{-6} \mathrm{mbar}\right)$ after completion of the dehydration process described in section 2.1. Prior to any other action, continuous reflectivity measurement at one wavelength inside a hydration band (either at 1.93 or $3.10 \mu \mathrm{m}$ ) as well as continuous measurements of pressure, sample temperature and room temperature are started. A given quantity of water vapor is produced in a known volume by evaporation from purified and thoroughly degassed liquid water. Then, the opening of a valve between this volume and the simulation chamber allows the adsorption of water vapor onto the sample. When equilibrium is reached (no significant temporal evolution of pressure and reflectance), continuous reflectance measurement at one wavelength is stopped and a full reflectance spectrum is measured. After completion of the full spectrum measurement, the full procedure is repeated to obtain measurements for a new hydration step. The procedure is repeated until the pressure at equilibrium after adsorption on the sample reaches a value corresponding to a relative humidity of about $75 \%$. This limitation in terms of relative humidity is imposed by the coldest point in the simulation chamber that constantly displays a temperature $3 \mathrm{~K}$ lower than the sample itself.

\subsection{Calculation of Water Content}

[14] Near-infrared reflectance spectroscopy is used to determine the amount of water adsorbed in the sample at each step of the hydration/dehydration process. As the relationship between the amount of adsorbed water and the strength of the spectral criteria is sample-dependent, we have to determine this empirical relationship for each sample. This is done by comparing the strength of the spectral criteria with the amounts of water introduced in the chamber for the first adsorption steps at low relative pressure. We carefully checked that under these conditions of low relative 
Smectite (SWy-2)
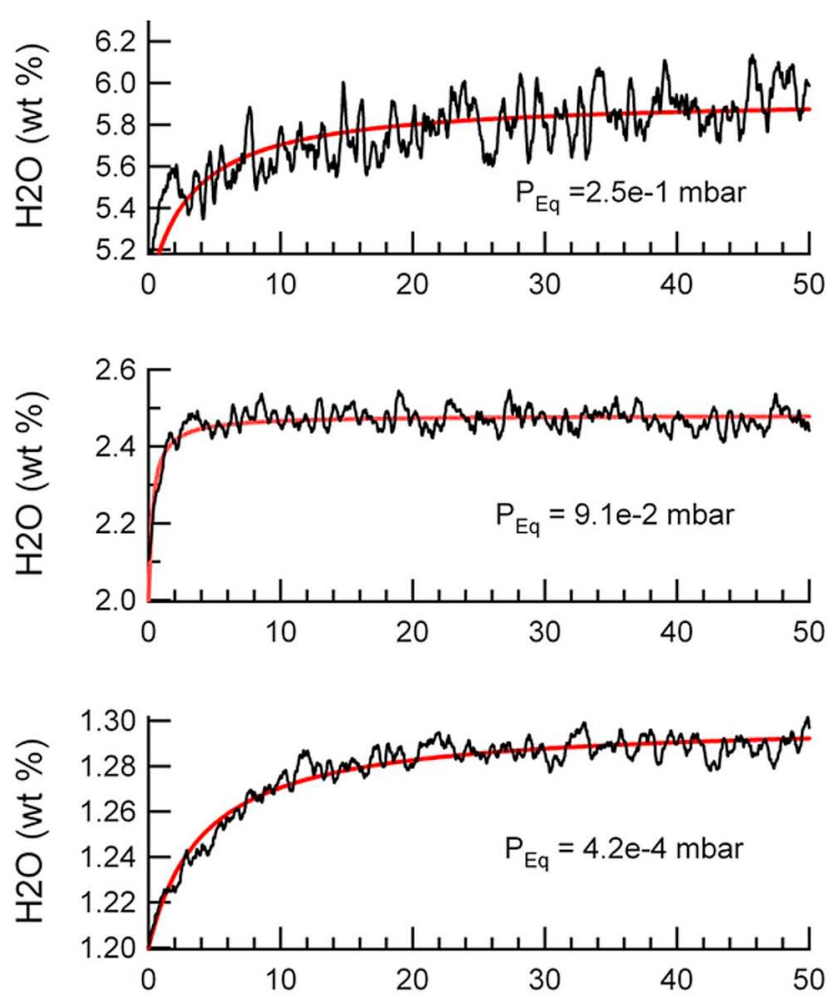

Time (min)

Figure 3. Evolution of the water content of the sample as a function of time for different water injections, in the case of the smectite sample (black curve) (The indicated pressure corresponds to the vapor pressure after the sample has equilibrated.) Fit to the curves obtained from equation (5) (red curve). Because water contents were determined through spectroscopy, they might be slightly overestimated due to the presence of $-\mathrm{OH}$ groups.

pressure, the amount of water adsorbed on surfaces of the chamber other than the sample itself remains low enough to not affect our calculations. This is not true at higher relative pressure, thus justifying the need for another method as reflectance spectroscopy [Pommerol et al., 2009].

[15] Because the wavelengths at which spectral criteria are calculated correspond to high levels of absorption (low reflectance values), only a few grains are involved in the reflection process [Douté and Schmitt, 1998]. By monitoring the behavior of reflectance inside one of those two hydrations bands, we are able to follow the water content of the very top surface of the sample (a few tens of microns).

\section{Results}

\subsection{Isotherms}

[16] The isotherms obtained have been described elsewhere [Pommerol et al., 2009] and will not be discussed in detail. For each material, these isotherms were fitted suc- cessfully according to the Langmuir theory [Gregg and Sing, 1982] (Figure 2). While in previous work we used the BET description for adsorption isotherms, the choice of the Langmuir theory resided in the fact that it can be easily connected to a kinetics theory. The quality of the Langmuir isotherm fit to our water adsorption data is slightly less good than using the BET theory, which better take into account multilayer adsorption, but it is still reasonable considering our error on absolute water contents and relative pressure measurements (10\% on both). It is important to note that the two theories are equivalent at low pressure, where only a monolayer is expected to be present on the mineral surfaces. According to the Langmuir theory the amount of water $\rho_{a}$ that adsorbs at a given pressure $P$ is

$$
\rho_{a}=C \frac{\alpha P}{1+\alpha P}
$$

The $C$ constant "scales" the amount of water that can be adsorbed at a given pressure (it is related to the specific surface area), while the alpha parameter controls the curvature of the isotherm and is related to the adsorption energy. Our estimated values of $C$ and $\alpha$ are reported in Table 1.

[17] The amounts of water adsorbed on the surface as a function of time for the different hydration steps are presented in Figures 3 and 4. Those measurements show a variability of behaviors among the different samples as well as an evolution of their behavior for increasing relative humidity. The time scale for the adsorption process to reach equilibrium ranges from a few tens of seconds to a few tens of minutes for the samples studied.

\subsection{Evidences for Diffusion}

[18] By comparing our estimates of the water content of the sample upper surface layer with the global amount of water adsorbed by the sample, we obtained strong evidence for a global equilibrium time lag due to water diffusion through our thin sample $(1 \mathrm{~mm})$ (Figure 5). As an example, the ferrihydrite sample shows a nonmonotonic behavior of its surface water content that presents an almost instantaneous increase followed by a slow decrease toward an equilibrium value. We interpret this behavior as an effect of the slow diffusion of the water from the top to the bottom of the sample: as a response to the initial increase in vapor pressure of the chamber, only the sample upper surface is adsorbing water. Then vapor desorbs from the surface grains and diffuse toward the lower sample layers. Such vertical gradients of adsorbed water are only visible for the two samples that adsorb the largest quantities of water: ferrihydrite and JSC-Mars1. Therefore, it is crucial to use either very thin samples $(<100 \mu \mathrm{m})$ or a surface proxy for water

Table 1. Fit Parameters for Langmuir Isotherm Model of the Adsorption Isotherms

\begin{tabular}{lccccc}
\hline & JSC1 & Ferri & Dunite & Tuff & Smectite \\
\hline Alpha $\left(\mathrm{P}^{-1}\right)$ & 0.81 & 0.45 & 0.17 & 0.70 & 0.21 \\
$1 \sigma$ & 0.18 & 0.09 & 0.04 & 0.16 & 0.1 \\
$\mathrm{C}\left(\mathrm{kg} \mathrm{m}^{-3}\right)$ & 55.9 & 83.8 & 2.0 & 6.3 & 10.7 \\
$1 \sigma$ & 2.854 & 4.347 & 0.387 & 0.321 & 0.430 \\
\hline
\end{tabular}




\section{Ferrihydrite}
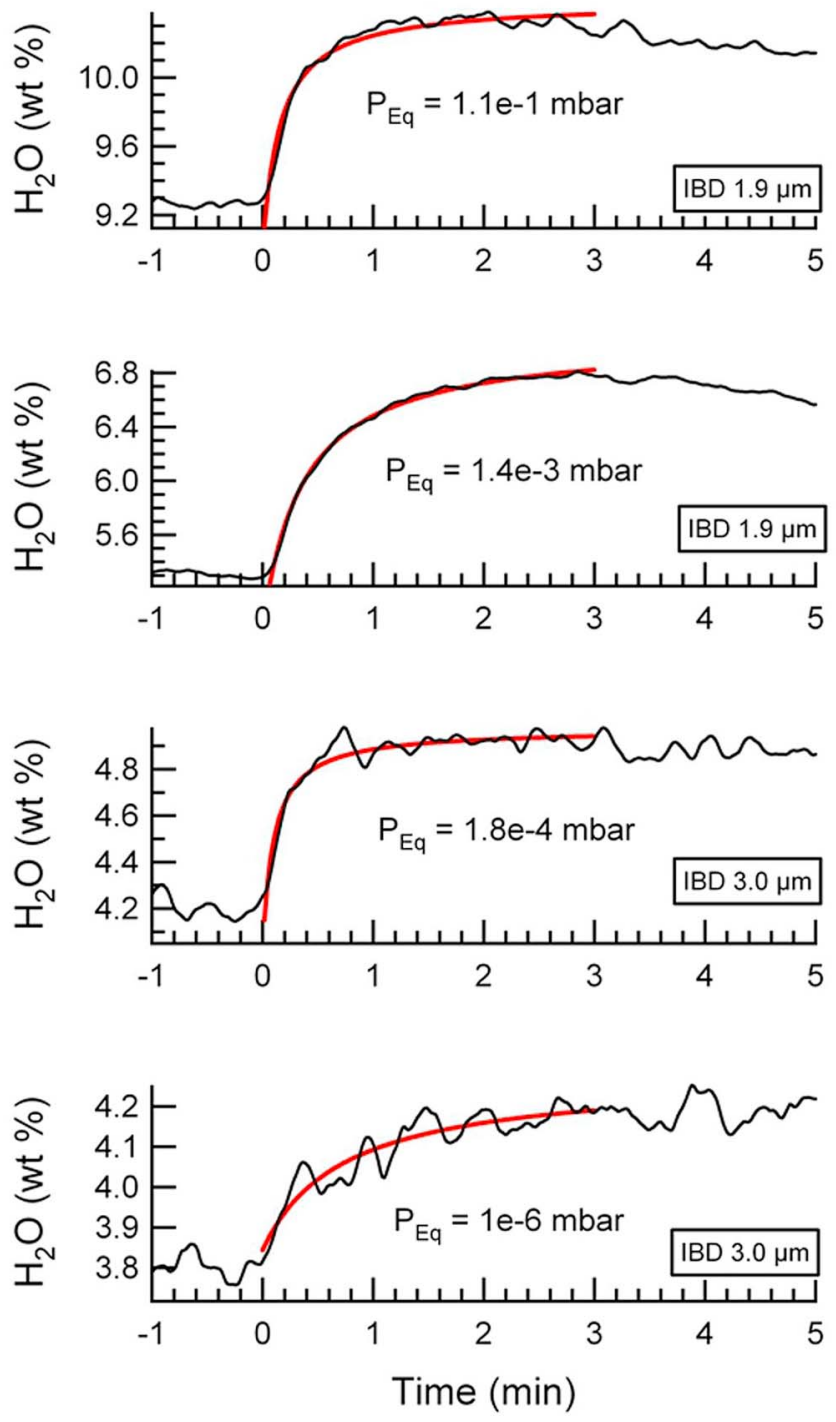

Figure 4. Evolution of the water content of the sample as a function of time for different water injections, in the case of the ferrihydrite sample (black curve). (The indicated pressure corresponds to the vapor pressure after the sample has equilibrated.) Fit to the curves obtained from equation (5) (red curve). Because water contents were determined through spectroscopy, they might be slightly overestimated due to the presence of $-\mathrm{OH}$ groups. Refer to Figure 1 for absolute water contents.

content (spectroscopy in our case) to obtain kinetics values that are not biased by diffusion within the sample.

\subsection{Kinetics}

[19] According to the Langmuir theory, the adsorption kinetics can be formalized as follows. The variation of the surface coverage $(\theta)$ as a function of time is the sum of the amount of water molecules that stick on the material minus the amount of molecules that desorbs:

$$
\frac{d \theta}{d t}=k_{a}(1-\theta)-k_{d} \theta
$$

where $k_{a}$ and $k_{d}$ are the kinetics constants for adsorption and desorption, respectively. They can be written as $[D o, 1998]$

$$
k_{a}=C s \frac{P}{\sqrt{2 \pi M k T}}
$$

$C s$ is the sticking coefficient, $M$ is the mass of a water molecule, and $k$ is the Boltzmann constant.

$$
k_{d}=k_{d \infty} e^{-\frac{E_{d e s}}{R T}}
$$

$k_{d \infty}$ is a preexponential factor that could be considered as the "jump attempt frequency," $\nu$, at overcoming the desorption barrier, and $E_{d e s}$ is the molar heat of desorption.

[20] For the successive adsorption steps performed on the sample, integration of equation (4) leads to

$$
C(t)=C_{i}+\left(C_{i+1}-C_{i}\right)\left(1-\exp \left(-k_{t o t} t\right)\right)
$$

where $C(t)$ is the amount of water adsorbed on the sample (wt\%), $C_{i}$ is the amount of water before the adsorption step, $C_{i+1}$ is the amount of water adsorbed when equilibrium is reached, and $k_{t o t}$ is the global kinetics constant that equals

$$
k_{\text {tot }}=k_{a}+k_{d}=(1+\alpha P) k_{d}
$$

For each of the adsorption steps, the measured $C(t)$ curves were fitted using equation (5), in order to obtain $k_{t o t}$ Then, $k_{a}$ and $k_{d}$ were extracted using equation (6). The value of alpha has been previously obtained from the measured isotherm. Results of this analysis are presented in Figures 6 and 7 for the different samples.

[21] It is crucial to remark that $k_{a}$ increases with $P$ while $k_{d}$ is independent of pressure. Thus, as pressure decreases, adsorption kinetics becomes controlled by $k_{d}$. Such behavior is clearly seen in the case of the smectite sample. At low

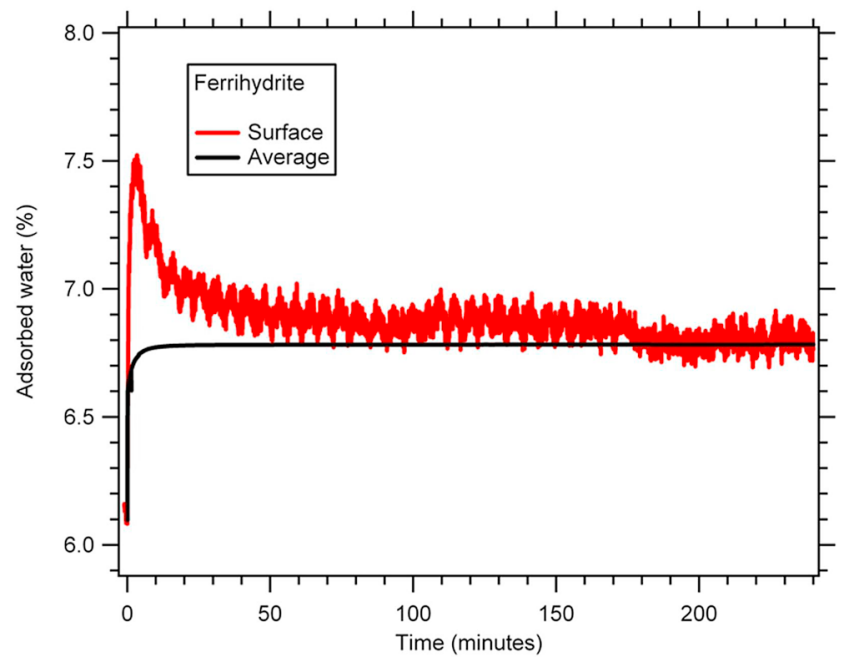

Figure 5. Evolution of the average water content of the ferrihydrite sample (black curve, estimated from volumetry) and the surface hydration at the very top of the sample (red curve) determined from spectroscopy. The surface behavior is decoupled from the bulk sample behavior, which shows evidence of the presence of water diffusion through the sample. 
pressure, $k_{t o t}$ is constant, while for pressures in excess of 2-3 Pa, $k_{\text {tot }}$ begins to increase with $P$, which corresponds to the onset of an adsorption control on the kinetics process. Calculations of $k_{a}$ and $k_{d}$ using the value of $\alpha$ obtained from the isotherm reveal the expected behavior. For water vapor partial pressures below $10 \mathrm{~Pa}, k_{d}$ is constant among the
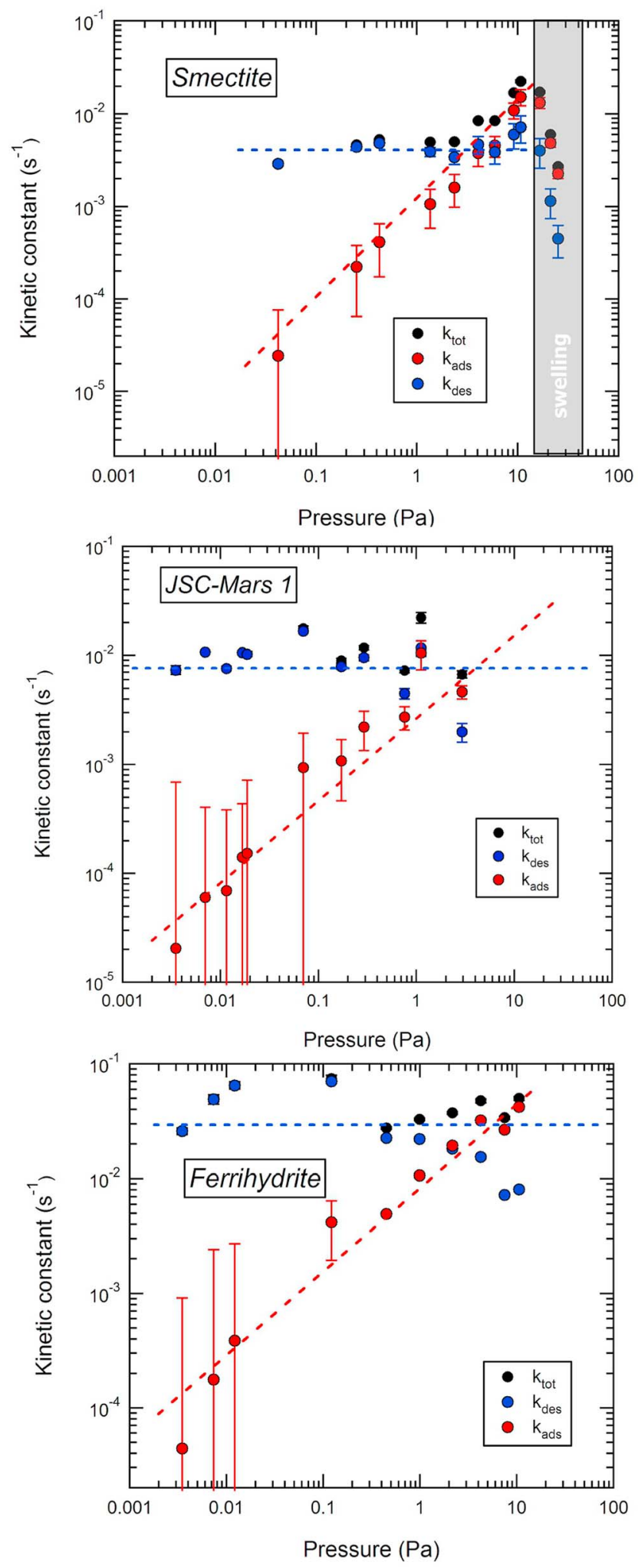

pressure range we studied $\left(k_{d}=0.21 \pm 0.02 \mathrm{~min}^{-1}\right)$, while $k_{a}$ shows a linear increase with pressure. Both the equilibrium and kinetics approach are consistent. For pressures above $10 \mathrm{~Pa}$, smectite swelling begins which slows down the adsorption process and invalidates the Langmuir theory for this pressure range.

[22] Values for $k_{d}$ vary between $3.0310^{-2} \mathrm{~s}^{-1}(1 / \mathrm{k}=33 \mathrm{~s})$ for the ferrihydrite down to $k_{d}=3.510^{-3} \mathrm{~s}^{-1}$ for the smectite sample $(1 / \mathrm{k}=285 \mathrm{~s})$ (Table 2). The pressure derivative of $k_{a}$ shows variability among the samples studied, with values ranging from $4.3410^{-3} \mathrm{~s}^{-1} \mathrm{~Pa}^{-1}$ to $1.4710^{-4} \mathrm{~s}^{-1} \mathrm{~Pa}^{-1}$ with the exception of the dunite sample which presents a significantly lower value $1.4710^{-4} \mathrm{~s}^{-1} \mathrm{~Pa}^{-1}$. The values we have measured here for $k_{a}$ and $k_{d}$ are significantly higher than previously reported in the literature for Martian analogs. However, these previous estimates also showed strong variability. Zent et al. [2001] reported a value of $\sim 5.10^{-6} \mathrm{~s}^{-1}$ for $k_{a}$ in the case of the SWy-1 smectite $(P=62 \mathrm{~Pa}, T=273 \mathrm{~K})$ while Chevrier et al. [2008] found a value of $2 \cdot 5 \cdot 10^{-4} \mathrm{~s}^{-1}$ for a montmorillonite-type smectite under similar conditions $(P=57 \mathrm{~Pa}, T=270 \mathrm{~K})$. However, our values are consistent with values of $k_{d}$ reported for minerals dust $\left(k_{d}=\right.$ $1.10^{-3}$ at $265 \mathrm{~K}$ ) [Seisel et al., 2005]. The kinetics time scales we measure reveal significantly faster equilibration than previously estimated for the Martian surface. We attribute this difference to the use of large samples by previous researchers and the resulting limitation of adsorption by diffusion in previous studies that leaded to a significant slow down of the process.

\subsection{Temperature Dependence of $\boldsymbol{k}_{a}$ and $\boldsymbol{k}_{d}$}

[23] Our experiments were performed at a single temperature of $243 \mathrm{~K}$. Seasonal and diurnal temperature variations are important on Mars, and some hypothesis on the temperature dependence of $k_{a}$ and $k_{d}$ is required if one wants to extrapolate our results.

[24] In the case of the desorption process, the molecule can be seen as oscillating in a potential well and thus the temperature dependence of desorption is expected to follow an Arrhenius type law (equation (4)). Molar heat of desorption can be determined by differential scanning calorimetry. Values found in the literature for clay minerals are of $63.3 \mathrm{~kJ} \mathrm{~mol}^{-1}$ for nontronite and $64.6 \mathrm{~kJ} \mathrm{~mol}^{-1}$ for montmorillonite [Janchen et al., 2006], which were obtained under a relative pressure of 0.3 .

[25] The microphysics of adsorption appears more complex. The adsorption of a water molecule is controlled by the rate at which molecules collide on the surface and the ability of a colliding molecule to stick to the surface (exchange

Figure 6. Evolution of the adsorption kinetics constant $\left(k_{a d s}\right)$ and the desorption kinetics constant $\left(k_{d e s}\right)$ as a function of pressure for the smectite, JSC-Mars1, and ferrihydrite samples. The values of $k_{a d s}$ and $k_{d e s}$ were obtained by combining $k_{t o t}=k_{a d s}+k_{d e s}$ and the Langmuir isotherm parameters. The combined decrease of $k_{a d s}$ and $k_{\text {des }}$ at elevated pressure for the smectite sample is likely due to onset of interlayer swelling. Because water contents were determined though spectroscopy, they might be slightly overestimated due to the presence of $-\mathrm{OH}$ groups. Refer to Figure 1 for absolute water contents. 

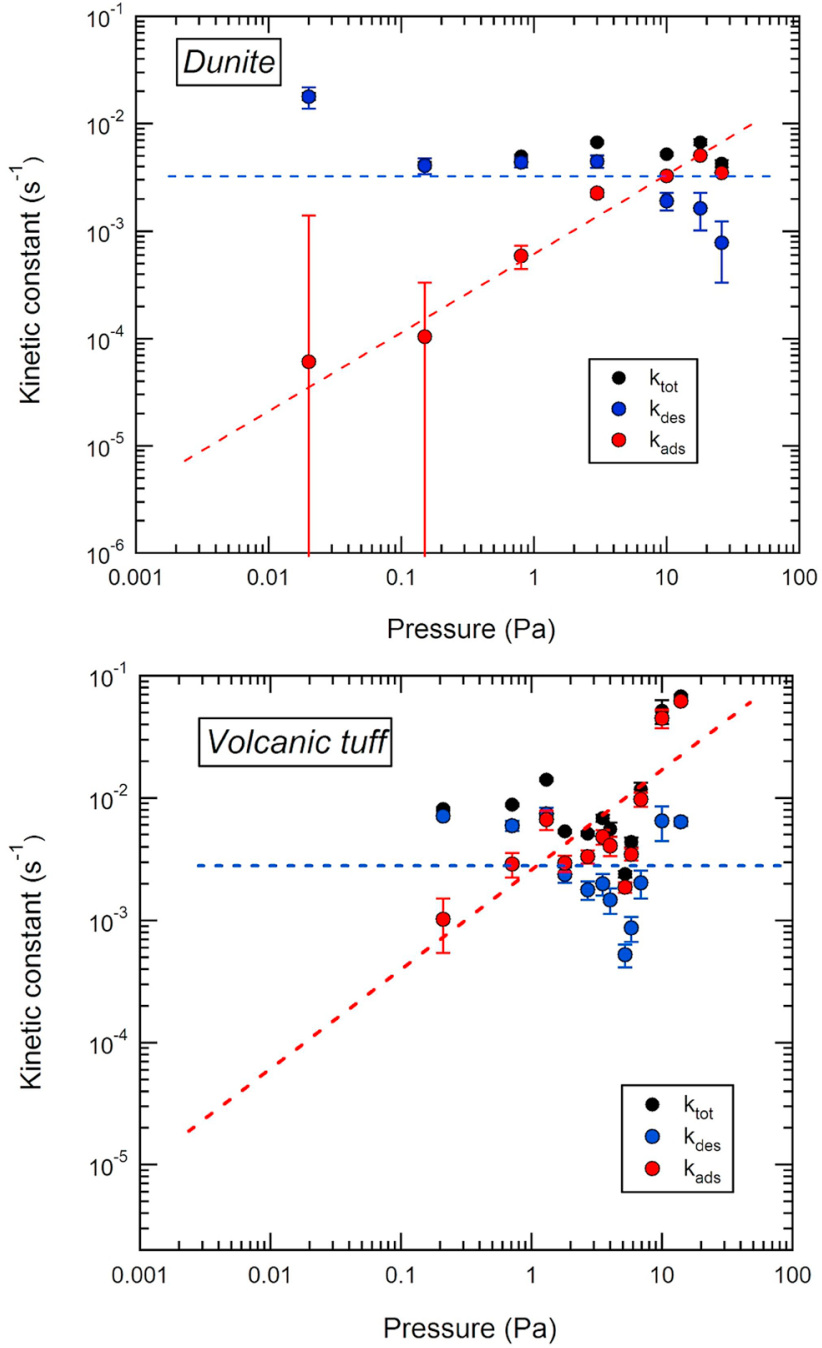

Figure 7. Evolution of the adsorption kinetics constant $\left(k_{a d s}\right)$ and the desorption kinetics constant $\left(k_{d e s}\right)$ as a function of pressure for the dunite and volcanic tuff samples. The values of $k_{a d s}$ and $k_{d e s}$ were obtained by combining $k_{t o t}=k_{a d s}+k_{d e s}$ and the Langmuir isotherm parameters.

momentum and then fall in the potential well). The collision rate can be obtained from kinetic gas theory as

$$
\frac{P}{\sqrt{2 \pi M k T}}
$$

and then depends on temperature. A sticking coefficient $C s$ is commonly defined that takes into account the ability of molecule to stick to the surface. This coefficient and its temperature dependence can hardly be estimated by simple considerations since it requires the understanding of the fine

Table 2. Adsorption Kinetics Parameters for the Various Samples Studied

\begin{tabular}{lccccc}
\hline Sample & JSC1 & Ferrihydrite & Smectite & Tuff & Dunite \\
\hline$k_{d}\left(\mathrm{~s}^{-1}\right)$ & $9.010^{-3}$ & $3.0310^{-2}$ & $3.510^{-3}$ & $3.6710^{-3}$ & $5.010^{-3}$ \\
$1 \sigma\left(\mathrm{s}^{-1}\right)$ & $1.210^{-3}$ & $7.110^{-3}$ & $3.310^{-4}$ & $6.710^{-4}$ & $2.210^{-3}$ \\
$d k_{a} / d P\left(\mathrm{~s}^{-1} \mathrm{~Pa}^{-1}\right)$ & $2.7010^{-3}$ & $4.3410^{-3}$ & $1.4710^{-3}$ & $3.3010^{-3}$ & $2.010^{-4}$ \\
\hline
\end{tabular}

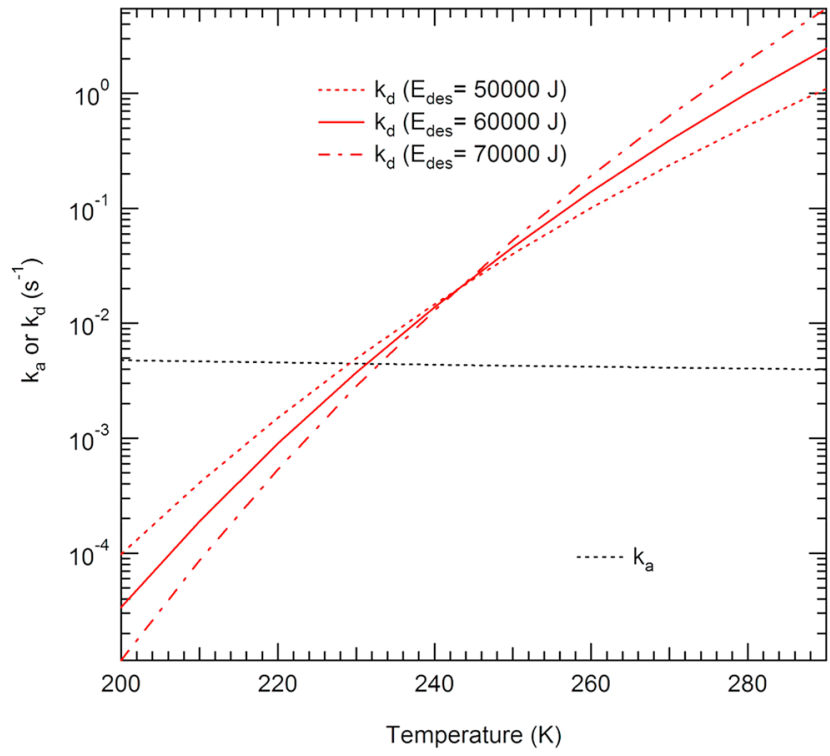

Figure 8. Expected temperature dependence of $k_{a}$ and $k_{d}$ in the case of the ferrihydrite sample, extrapolated from our laboratory measurements $(P=1 \mathrm{~Pa}, T=243 \mathrm{~K})$.

energy transfer between the colliding molecule and the surface [Zangwill, 1988]. Experimental investigation of water ice condensation can however provide some information from measurements of the temperature dependence of the condensation coefficient. The condensation coefficient is equivalent to the sticking coefficient, in the case where a gas molecule collides with the corresponding molecular solid. Values of the condensation coefficient of water were found to decrease by a factor of three in the $140 \mathrm{~K}-$ $250 \mathrm{~K}$ temperature range (see compilation by Kossacki et al. [1999]). Because this temperature dependence of the sticking coefficient appears moderate with regard to diurnal variations, it will be neglected in the rest of the analysis.

[26] Using the analytical forms of $k_{a}$ and $k_{d}$, together with a set of plausible values for the heat of desorption, we have calculated the effect of temperature on $k_{a}$ and $k_{d}$ at a pressure of $1 \mathrm{~Pa}$ (Figure 8). Calculations for $k_{d}$ were performed using values of 50,60 , and $70 \mathrm{~kJ} \mathrm{~mol}^{-1}$ for the heat of desorption. The calculations show that $k_{d}$ can vary by 4 orders of magnitude over the $200 \mathrm{~K}-270 \mathrm{~K}$ temperature range. A temperature dependence of $k_{a}$ is also present (related to the $T^{-1 / 2}$ dependence of the collision frequency) but the decrease is less than $20 \%$ across the $200 \mathrm{~K}-270 \mathrm{~K}$ range. It is important to note that the time scale for adsorption is controlled by

$$
\tau=\frac{1}{k_{a}+k_{d}}
$$

Therefore at low temperature, the temperature dependence of $k_{a}$ is expected to be minor, and the kinetic of the absorption process will be controlled by $k_{d}$ only.

\section{Discussion}

\subsection{Vapor Transport in a Porous Nonadsorbing Media}

[27] Atmospheric water molecules will be transported deep into the regolith by transport in the gas phase, within 
porosity. At this point we neglect the presence of surface diffusion (diffusion of adsorbed water molecules at the surface of grains) for which little is known and can be hardly constrained. We suppose that vapor transport only takes place in the gas phase for which the water transport is a diffusive process controlled by a diffusion equation:

$$
\frac{\partial \rho}{\partial t}=D_{\text {micro }} \frac{\phi}{\tau} \Delta \rho
$$

where $\phi$ and $\tau$ are geometrical parameters that are porosity and tortuosity, respectively. The diffusion coefficient $D_{\text {micro }}$ $\left(\mathrm{m}^{2} \mathrm{~s}^{-1}\right)$ is the parameter that characterizes the transfer rate at the microscopic scale. Because tortuosity can be difficult to quantify for a natural sample, one might define a diffusion coefficient at the "mesoscopic" scale (or in soil diffusion coefficient) that can be directly measured experimentally:

$$
D_{\text {meso }}=D_{\text {micro }} \frac{\phi}{\tau}
$$

In a porous media, three diffusion regimes can be distinguished that are the Fickian, Knudsen, and intermediate regime, for which the diffusion coefficient will change.

[28] In the Fickian regime (of diffusion coefficient $D_{\text {micro }}=$ $\left.D_{\text {Fickian }}\right)$, diffusion is controlled by collision between gas molecules (with $\mathrm{H}_{2} \mathrm{O}$, and also $\mathrm{CO}_{2}$ for the Martian atmosphere), and thus relates to pressure and temperature. Analysis using a hard sphere model shows that it should scale with $T^{3 / 2}$, which is in fair agreement with measurements of water diffusion within $\mathrm{CO}_{2}$ [Hudson et al., 2007].

[29] At low pressure, collisions between molecules are less frequent, and the mean molecular free path is increased. When the mean molecular free path is above the typical pore diameter in the soil, the diffusion will be controlled by the geometry of the void space, and the Knudsen regime has now to be considered with diffusion coefficient $D_{\text {micro }}=$ $D_{\text {Knudsen. }}$ In this regime, the presence of various gas phases does not affect the diffusion coefficient, since interspecies collisions are extremely rare.

[30] Calculation of the water molecule mean free path for $T=200 \mathrm{~K}$ and $P=600 \mathrm{~Pa}$ (Mars typical surface pressure) results in a value of the order of $10 \mu \mathrm{m}$. Because this value is of the order of the expected pore size for Mars surface dust one might consider the intermediate regime in which both collisions with pore walls and intermolecular collisions are important. In this regime the diffusion coefficient is obtained as

$$
\frac{1}{D_{\text {meso }}}=\frac{1}{D_{\text {Fickian }}}+\frac{1}{D_{\text {Knudsen }}}
$$

\subsection{Vapor Transport in a Porous Adsorbing Media}

[31] In all the Fickian, Knudsen, and intermediate regimes, the water molecule transfer rate is controlled by vapor density gradient. Through adsorption, water molecules will be removed from the gas phase to an adsorbed state, which will significantly reduce vapor gradient, and then slow down the transport process.
[32] A macroscopic diffusion coefficient, $D_{\text {micro }}$, smaller than in the nonadsorbing case, can then be defined as [Schorghofer and Aharonson, 2005]

$$
D_{\text {macro }}=D_{\text {meso }} \frac{1}{1+\frac{R T}{18.10^{-3}} \frac{\partial \rho_{a}}{\partial P}}
$$

where $\frac{\partial \rho_{a}}{\partial P}$ is the pressure derivative of the amount of water adsorbed per mass unit $\left(\mathrm{kg} \mathrm{kg}^{-1}\right)$. Equation (10)) thus implies that in the case of an adsorbing regolith $\frac{\partial \rho_{a}}{\partial P}$, i.e., the slope of the isotherm curve $\left(\rho_{a}\right.$ as a function of $P$ ), will have an influence on the transfer. However, once a steady state is reached the adsorption properties will no longer influence the transfer [Schorghofer, 2007; Schorghofer and Aharonson, 2005].

[33] From the effective diffusion coefficient, it is possible to calculate an adsorption skin thickness as a response to the diurnal cycle by using

$$
L_{e q}=\sqrt{\frac{D_{\text {macro }}}{2 \nu}}
$$

where $v$ is the frequency corresponding to the Martian diurnal cycle $\left(1 /(24.66 * 3600)=1.126 \times 10^{-5} \mathrm{~Hz}\right)$. In the case of the Langmuir theory, this skin thickness can be expressed in terms of $C$ and $\alpha$ parameters as

$$
L_{e q}=\sqrt{\frac{1}{2 \nu} D_{\text {meso }} \frac{1}{1+\frac{R T}{18.10^{-3}} \frac{\alpha C}{(1+\alpha P)^{2}}}}
$$

Because the slowing factor in the denominator is large, this can be simplified to

$$
L_{e q}=\sqrt{\frac{18 \cdot 10^{-3}}{R T} D_{m e s o} \frac{1}{2 \nu} \frac{(1+\alpha P)^{2}}{\alpha C}}
$$

[34] At the macroscopic scale, the water molecules will travel within the regolith before reaching the adsorption equilibrium. This is a direct consequence of the intrinsic kinetics and the effect can be estimated as follows.

[35] The characteristic time of the adsorption phenomena

$$
\tau_{a d s}=\frac{1}{k_{t o t}}
$$

is the inverse of $k_{t o t}$. Before being adsorbed, the molecule will travel a distance $L_{k i n}$ in the soil that scales as

$$
L_{k i n}=\sqrt{D_{m e s o} \tau_{a d s}}=\sqrt{\frac{D_{\text {meso }}}{k_{\text {tot }}}}
$$

[36] Calculations of the skin thicknesses in the equilibrium and kinetics adsorption cases are plotted on Figure 9, using our measured values of C, $\alpha, k_{a}$ and $k_{d}$ at $243 \mathrm{~K}$ for the five samples studied. For the equilibrium case, the adsorption skin thickness ranges between a few $\mu \mathrm{m}$ at low pressure to at maximum $2 \mathrm{~mm}$ for a partial pressure of $100 \mathrm{~Pa}$ 


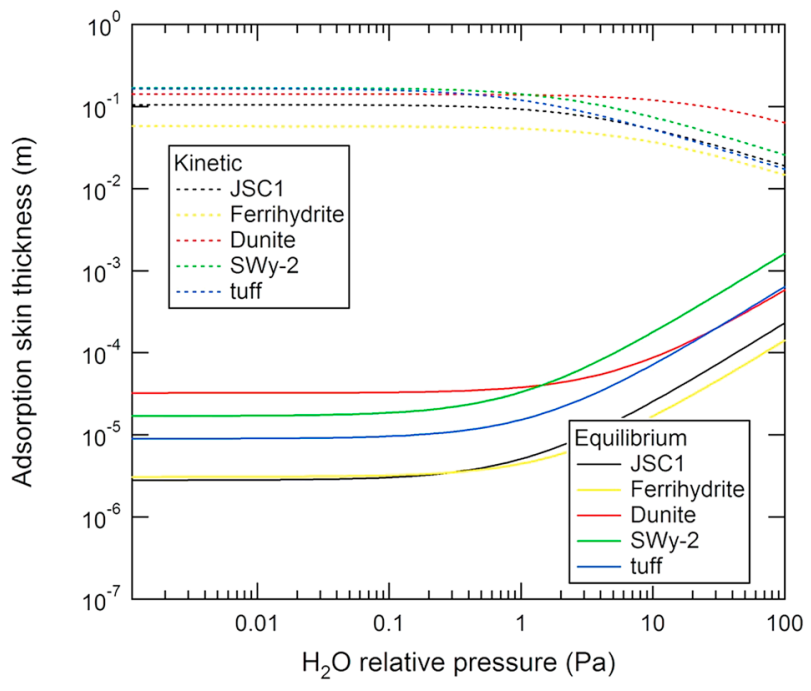

Figure 9. Calculated adsorption skin thickness as a function of water partial pressure according to the equilibrium and kinetics theory.

(which is unrealistically high for the water partial pressure on Mars). The introduction of a kinetics dependence of adsorption strongly increases the skin thickness. Values found using equation (14) range between a few centimeters at high pressure to $\sim 15 \mathrm{~cm}$ at low pressure (Figure 9). This latter value is of the order of the diurnal thermal skin thickness that controls the diurnal adsorption cycle in the regolith. Consequently intense regolith breathing might be expected. We also performed these calculations for 2 extreme temperatures, $200 \mathrm{~K}$ and $270 \mathrm{~K}$ of the daily cycle to estimate possible strong nonlinear effects of $k_{d}$ on skin depth (using $E_{\text {des }}=60 \mathrm{~kJ} \mathrm{~mol}^{-1}$ ). The calculations show that the effect of temperature is to change the skin depth at low pressure by about a factor of 3 relative to our value at $243 \mathrm{~K}(\sim 14 \mathrm{~cm}$ at $200 \mathrm{~K}$ and $\sim 1.7 \mathrm{~cm}$ at $270 \mathrm{~K}$ in the case of the ferrihydrite sample at $1 \mathrm{~Pa}$ ), which imply that temperature does not effect our results qualitatively.

[37] From the skin thickness and using a Langmuir isotherm, the maximum amount of exchangeable water per square meter of soil can be estimated from

$$
m=\rho_{a d s} L=C \frac{\alpha P}{1+\alpha P} L\left(\text { in } \mathrm{kg} \mathrm{m}^{-2}\right)
$$

For the equilibrium and kinetics cases this results in

$$
\begin{gathered}
m_{e q}=C \frac{\alpha P}{1+\alpha P} \sqrt{\frac{18.10^{-3}}{R T} D_{\text {meso }} \frac{1}{2 \nu} \frac{(1+\alpha P)^{2}}{\alpha C}} \\
m_{\text {kin }}=C \frac{\alpha P}{1+\alpha P} \sqrt{\frac{D_{\text {meso }}}{k_{\text {tot }}}}
\end{gathered}
$$

[38] Calculations were performed for $m_{e q}$ and $m_{k i n}$, and results are expressed in precipitable $\mu \mathrm{m}\left(m_{p p \mu}=m \rho_{H 20} 10^{6}\right.$ where $\rho_{H 20}$ is the density of water $\left.\left(\sim 1000 \mathrm{~kg} \mathrm{~m}^{-3}\right)\right)$ using a value of $10^{-4} \mathrm{~m}^{2} \mathrm{~s}^{-1}$ for $D_{\text {meso }}$ (the value found by Chevrier et al. [2008], and a lower bound of the results of Hudson et al. [2007]). The estimates shown in Figure 10 suggest that the maximum amount of exchangeable water considerably increases when taking into account the intrinsic adsorption kinetics. For a water pressure of $\sim 0.1 \mathrm{~Pa}$ the amount of exchangeable water is between $10^{-3}$ to $10^{-2}$ precipitable $\mu \mathrm{m}$ while in the kinetics case, it ranges between a few to several hundreds microns depending on the soil adsorption properties. The effect of temperature is to change this maximum amount by about a factor of 3 relative to our value at $243 \mathrm{~K}$ $(\sim 3750 \mu \mathrm{m}$ at $200 \mathrm{~K}$ and $\sim 445 \mathrm{pp} \mu \mathrm{m}$ at $270 \mathrm{~K}$, in the case of the ferrihydrite sample at $1 \mathrm{~Pa}$ ).

[39] We should stress that the estimates presented in Figure 10 are most probably overestimated. We suppose that the regolith equilibrates with a $0.1 \mathrm{~Pa}$ partial water pressure, and that there is no limitation in the amount of vapor available. In addition, a more accurate modeling that includes combined daily temperature and pressure variations needs to be undertaken. However, these high values are likely to contribute to explain the significant variations observed in Mars's atmospheric humidity during the course of the day, if the atmospheric mixing is sufficiently efficient.

[40] Our experimental study has been performed at a single temperature of $243 \mathrm{~K}$. This temperature is encountered during Mars summertime at equatorial to tropical latitude. In order to extrapolate our results to lower temperatures, the knowledge of the temperature dependence of $k_{a}$ and $k_{d}$ is required. At high water vapor pressure $(\alpha P>1)$ the kinetics will be controlled by $k_{a}$ which scales with the square root of temperature. In this pressure regime, only a moderate temperature dependence is expected. At low pressure $(\alpha P<1)$,

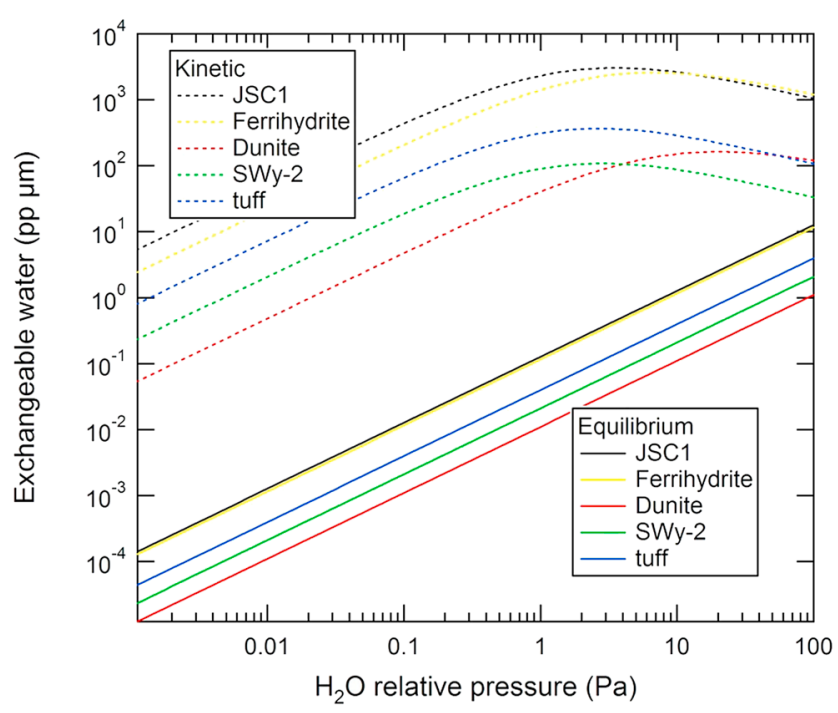

Figure 10. Amount of exchangeable water during the diurnal cycle as a function of water partial pressure for the various samples studied. These values are the maximum amount of water that can be exchanged during the course of the day and illustrate the influence of the surface geological material on the daily water cycle. 
there is an exponential dependence of $k_{d}$ on temperature and a potentially important effect of $T$.

[41] There are some differences in the amount of exchangeable water among the samples, which may imply some geological control on the diurnal variations of surface humidity. The dunite and smectite samples show the smaller amount of exchangeable water while the JSC-Mars1 and ferrihydrite sample have high and similar values. The last two samples also have remarkably similar isotherms [Pommerol et al., 2009]. X-ray diffraction analysis we performed on the JSC-Mars1 samples suggest the presence of a minor amount of ferrihydrite. Because of its strong adsorption capacity, this "mineral" component might control the adsorption property of JSC-Mars1 and explain the similarity of our experimental results for the two samples (both in the equilibrium and kinetics cases). The JSC-Mars1 is a palagonitic soil, a more advanced alteration product than the dunite (nonaltered) or the tuff. In the later, ferrihydrite is absent and iron is hosted in silicates and anhydrous iron oxide.

[42] There are some hints for a surface geological impact on the Mars atmospheric water cycle. An intriguing correlation is present on Mars between the surface thermal inertia and the average annual water vapor content of the atmosphere [Putzig and Mellon, 2007; Smith, 2002]. This correlation strongly points toward a possible coupling between the atmosphere and the subsurface. We favor adsorption as the coupling mechanism. The identification of nanophase iron hydroxyde on Mars [Morris et al., 2006], which have particular adsorption properties [Pommerol et al., 2009], strengthens this hypothesis. The adsorption capacity of ferrihydrite is extreme and the kinetics is fast with regard to the diurnal cycle period. The adsorption behavior of this iron oxyhydroxide is different from a pure oxide, since it has the ability to build hydrogen bonds with water molecules. If the Martian dust contains some amount of iron hydroxide, with ferrihydrite-like adsorption properties, it is likely to adsorb a significant part of the water column vapor. As an example, a $2.5 \mathrm{~mm}$ thick layer of ferrihydrite can adsorb the equivalent of $10 \mathrm{pp} \mu \mathrm{m}$ at a $\mathrm{H}_{2} \mathrm{O}$ vapor pressure of $0.1 \mathrm{~Pa}$ and $T=243 \mathrm{~K}$.

\section{Conclusion}

[43] We have measured the kinetics parameters $k_{a}$ and $k_{d}$ for mineral analogs of the Martian soils, at $P$ and $T$ conditions relevant for Mars' surface. We have shown that as well as the Langmuir isotherm parameter, the kinetics parameters vary among samples, which implies a geologic control on the atmosphere/surface coupling. The kinetics parameters are of the order $10^{-2}-10^{-3} \mathrm{~s}^{-1}(100-1000 \mathrm{~s})$, which is fast with regard to the diurnal cycle, but slow with regard to typical in soil vapor diffusion time scales. The presence of an intrinsic kinetics significantly increases the adsorption skin depth, i.e., the thickness of the surface layer that is involved in the diurnal cycle. Some water diffusion might also occur in the adsorbed state as surface grain diffusion but to date this process is not constrained.

[44] Among the samples, ferrihydrite, an iron oxyhydroxyde, presents a very large surface area and fast adsorption kinetics. The identification of oxyhydroxyde from IR spectroscopy and Landers, together with its extreme adsorption properties, suggest that it might be an active player in the current Mars water cycle. The kinetics parameters reported here along with thermodynamic parameters determined for the same samples by Pommerol et al. [2009] will be useful in future global and local modeling of the Martian water cycle.

[45] Acknowledgments. Thoughtful comments by two reviewers significantly helped improve the manuscript. CNES and the Programme National de Planétologie are acknowledged for financial support.

\section{References}

Bonnefoy, N. (2001), Développement d'un spectro-goniomètre pour l'étude de la réflectance bidirectionnel le des surfaces géophysiques: Application au soufre et perspectives pour le satellite Io, Ph.D. thesis, Université Joseph Fourier, Grenoble, France.

Bottger, H. M., S. R. Lewis, P. L. Read, and F. Forget (2005), The effects of the Martian regolith on GCM water cycle simulations, Icarus, 177(1), 174-189, doi:10.1016/j.icarus.2005.02.024

Brissaud, O., et al. (2004), Spectrogonio radiometer for the study of the bidirectional reflectance and polarization functions of planetary surfaces. 1. Design and tests, Appl. Opt., 43(9), 1926-1937, doi:10.1364/ AO.43.001926.

Bryson, K. L., V. Chevrier, D. W. G. Sears, and R. Ulrich (2008), Stability of ice on Mars and the water vapor diurnal cycle: Experimental study of the sublimation of ice through a fine-grained basaltic regolith, Icarus, 196(2), 446-458, doi:10.1016/j.icarus.2008.02.011.

Chevrier, V., D. R. Ostrowski, and D. W. G. Sears (2008), Experimental study of the sublimation of ice through an unconsolidated clay layer: Implications for the stability of ice on Mars and the possible diurnal variations in atmospheric water, Icarus, 196(2), 459-476, doi:10.1016/j. icarus.2008.03.009.

Do, D. D. (1998), Absorption Analysis: Equilibria and Kinetics, 892 pp., Imperial Coll. Press, London.

Douté, S., and B. Schmitt (1998), A multilayer bidirectional reflectance model for the analysis of planetary surface hyperspectral images at visible and near-infrared wavelengths, J. Geophys. Res., 103(E13), 31,36731,389, doi:10.1029/98JE01894.

Gregg, S. J., and K. S. W. Sing (1982), Adsorption, Surface Area and Porosity, 303 pp., Academic, New York.

Hudson, T. L., et al. (2007), Water vapor diffusion in Mars subsurface environments, J. Geophys. Res., 112, E05016, doi:10.1029/2006JE002815.

Jakosky, B. M., R. W. Zurek, and M. R. La Pointe (1988), The observed day-to-day variability of mars atmospheric water vapor, Icarus, 73(1), 80-90, doi:10.1016/0019-1035(88)90086-3.

Janchen, J., D. L. Bish, D. T. F. Mohlmann, and H. Stach (2006), Investigation of the water sorption properties of Mars-relevant micro- and mesoporous minerals, Icarus, 180(2), 353-358, doi:10.1016/j.icarus.2005.10.010.

Janchen, J., R. V. Morris, D. L. Bish, M. Janssen, and U. Hellwig (2009),

The $\mathrm{H}_{2} \mathrm{O}$ and $\mathrm{CO}_{2}$ adsorption properties of phyllosilicate-poor palagonitic dust and smectites under Martian environmental conditions, Icarus, 200(2), 463-467, doi:10.1016/j.icarus.2008.12.006.

Kossacki, K. J., W. J. Markiewicz, Y. Skorov, and N. I. Komle (1999), Sublimation coefficient of wawter ice under simulated cometary-like conditions, Planet. Space Sci., 47, 1521-1530, doi:10.1016/S00320633(99)00037-9.

Melchiorri, R., et al. (2009), OMEGA/Mars Express: South pole region, water vapor daily variability, Icarus, 201(1), 102-112, doi:10.1016/j. icarus.2008.12.018.

Morris, R. V., et al. (2006), Mössbauer mineralogy of rock, soil, and dust at Gusev crater, Mars: Spirit's journey through weakly altered olivine basalt on the plains and pervasively altered basalt in the Columbia Hills, J. Geophys. Res., 111, E02S13, doi:10.1029/2005JE002584.

Pommerol, A., and B. Schmitt (2008a), Strength of the $\mathrm{H}_{2} \mathrm{O}$ near-infrared absorption bands in hydrated minerals: Effects of measurement geometry, J. Geophys. Res., 113, E12008, doi:10.1029/2008JE003197.

Pommerol, A., and B. Schmitt (2008b), Strength of the $\mathrm{H}_{2} \mathrm{O}$ near-infrared absorption bands in hydrated minerals: Effects of particle size and correlation with albedo, J. Geophys. Res., 113, E10009, doi:10.1029/ 2007JE003069.

Pommerol, A., B. Schmitt, P. Beck, and O. Brissaud (2009), Water sorption on Martian regolith analogs: Thermodynamics and near-infrared reflectance spectroscopy, Icarus, 204, 114-136, doi:10.1016/j.icarus. 2009.06.013. 
Putzig, N. E., and M. T. Mellon (2007), Thermal behavior of horizontally mixed surfaces on Mars, Icarus, 191(1), 52-67.

Schorghofer, N. (2007), Theory of ground ice stability in sublimation environments, Phys. Rev. E, 75(4), 041201, doi:10.1103/PhysRevE. 75.041201.

Schorghofer, N., and O. Aharonson (2005), Stability and exchange of subsurface ice on Mars, J. Geophys. Res., 110, E05003, doi:10.1029/ 2004JE002350.

Seisel, S., A. Pashkova, Y. Lian, and R. Zellner (2005), Water uptake on mineral dust and soot: A fundamental view of the hydrophylicity of atmospheric particles?, Faraday Discuss., 130, 437-451, doi:10.1039/ b417449f.

Smith, M. D. (2002), The annual cycle of water vapor on Mars as observed by the Thermal Emission Spectrometer, J. Geophys. Res., 107(E11), 5115, doi:10.1029/2001JE001522.

Sprague, A. L., D. M. Hunten, L. R. Doose, and R. E. Hill (2003), Mars atmospheric water vapor abundance: 1996-1997, Icarus, 163(1), 88101, doi:10.1016/S0019-1035(03)00072-1.

Sprague, A. L., et al. (2006), Mars atmospheric water vapor abundance: 1991-1999, emphasis 1998-1999, Icarus, 184(2), 372-400, doi:10.1016/j.icarus.2006.05.021.
Zangwill, A. (1988), Physics at Surfaces, Cambridge Univ. press, Cambridge, U. K.

Zent, A. P., and R. C. Quinn (1995), Simultaneous adsorption of $\mathrm{CO}_{2}$ and $\mathrm{H}_{2} \mathrm{O}$ under Mars-like conditions and application to the evolution of the Martian climate, J. Geophys. Res., 100(E3), 5341-5349, doi:10.1029/ 94JE01899.

Zent, A. P., and R. C. Quinn (1997), Measurement of $\mathrm{H}_{2} \mathrm{O}$ adsorption under Mars-like conditions: Effects of adsorbent heterogeneity, J. Geophys. Res., 102(E4), 9085-9095, doi:10.1029/96JE03420.

Zent, A. P., R. M. Haberle, H. C. Houben, and B. M. Jakosky (1993), A coupled subsurface-boundary layer model of water on Mars, J. Geophys. Res., 98(E2), 3319-3337, doi:10.1029/92JE02805.

Zent, A. P., D. J. Howard, and R. C. Quinn (2001), $\mathrm{H}_{2} \mathrm{O}$ adsorption on smectites: Application to the diurnal variation of $\mathrm{H}_{2} \mathrm{O}$ in the Martian atmosphere, J. Geophys. Res., 106(E7), 14,667-14,674, doi:10.1029/ 2000JE001394.

P. Beck, O. Brissaud, A. Pommerol, and B. Schmitt, Laboratoire de planétologie de Grenoble, Université Joseph Fourier USOG, BP 53 , F-38041 Grenoble, CEDEX, France. (pierre.beck@obs.ujf-grenoble.fr) 